\title{
Quantum Confinement between Self-Organized Pt Nanowires on Ge(001)
}

\author{
Nuri Oncel, Arie van Houselt, Jeroen Huijben, Ann-Sofie Hallbäck, Oguzhan Gurlu, \\ Harold J. W. Zandvliet, ${ }^{*}$ and Bene Poelsema \\ Solid State Physics, MESA+Research Institute for Nanotechnology, University of Twente, P.O. Box 217, \\ 7500 AE Enschede, The Netherlands
}

(Received 30 March 2005; published 6 September 2005)

\begin{abstract}
The existence of one-dimensional (1D) electronic states between self-organized Pt nanowires spaced 1.6 or $2.4 \mathrm{~nm}$ apart on a $\mathrm{Ge}(001)$ surface is revealed by low-temperature scanning tunneling microscopy. These perfectly straight Pt nanowires act as barriers for a surface state (located just below the Fermi level) of the underlying terrace. The energy positions of the 1D electronic states are in good agreement with the energy levels of a quantum particle in a well. Spatial maps of the differential conductivity of the 1D electronic states conclusively reveal that these states are exclusively present in the troughs between the $\mathrm{Pt}$ nanowires.
\end{abstract}

PACS numbers: 73.22.-f, 68.37.Ef, 81.16.Dn

Self-organized one-dimensional materials have attracted quite some attention during the last decade. This interest is not only due to the relative ease with which these atomic scale structures can be produced, but also due to the wealth of exotic physical phenomena that these one-dimensional materials can exhibit. Among them are quantization of conductance, Peierls instability and charge density waves, magnetism, and Luttinger liquid behavior [1-7].

Much progress has been made in the fabrication and testing of devices incorporating two-dimensional electrons trapped in semiconductor heterostructures. This has allowed study of the physical properties of these artificial systems at length scales of the order of de Broglie wavelength of electrons [8,9]. Particularly beautiful experiments regarding the scattering and confinement of electrons on noble metal surfaces have been published by Crommie, Lutz, and Eigler [10,11], Bürgi et al. [12], Sánchez et al. [13] and $\mathrm{Li}$ et al. [14]. Another most interesting example is provided by the unoccupied, electron resonance states in monatomic $\mathrm{Au}$ chains assembled atom by atom on a $\mathrm{NiAl}(110)$ surface $[15,16]$. These kinds of experiments provide a powerful strategy for measuring the relation between geometric structure and electronic properties.

In most of these cases the measurements have been performed on individual man-made structures, with the advantage of a great variety of (mostly) two-dimensional structures. This approach has the inherent disadvantage of dealing with thermodynamically unstable structures. Here we make use of the spontaneous formation of perfect platinum $(\mathrm{Pt})$ nanowires on germanium $(\mathrm{Ge})(001)$ surfaces [17]. These wires constitute thermodynamically stable features and can be made homogeneously on a mesoscopic scale. The Pt nanowires have a cross section of only one atom, are literally defect and kink free, and have lengths up to hundreds of nanometers. They thus promise to satisfy a prerequisite for the further confinement of surface states in an additional dimension leading to a truly one-dimensional electron gas. The fact that the Pt atoms are ordered so well on $\mathrm{Ge}(001)$ enables us to image the quantum mechanical interference of surface state electrons within these selforganized atomic scale structures. The spectroscopic capabilities of the scanning tunneling microscope (STM) allow the observation of the spatial distribution of a preselected electronic state. Exploiting these combined favorable features we have been able to unveil and explore these novel electronic states, surprisingly confined between the $\mathrm{Pt}$ nanowires, rather than inside them, as shown below.

Experiments were performed with a scanning tunneling microscope operating in ultrahigh vacuum. Ge(001) surfaces were cut from nominally flat 3 in. by $0.5 \mathrm{~mm}$, singleside-polished $n$-type wafers. Samples were mounted on Mo holders and contact of the samples to any other metal during preparation and experiment were carefully avoided. The $\mathrm{Ge}(001)$ samples were cleaned by $800 \mathrm{eV} \mathrm{Ar}^{+}$ion sputtering and annealing at $1100( \pm 25) \mathrm{K}$. After several cleaning cycles the $\mathrm{Ge}(001)$ samples were atomically clean and exhibited a well ordered $(2 \times 1) / c(4 \times 2)$ domain pattern [18-20]. Subsequently, an equivalent of 0.25 monolayer of platinum was deposited onto the surface at room temperature. Platinum was evaporated by resistively heating a $W$ wire wrapped with high purity $\mathrm{Pt}(99.995 \%)$. After $\mathrm{Pt}$ deposition the sample was annealed at $1050( \pm 25) \mathrm{K}$ for $10 \mathrm{~min}$. and then cooled down to room temperature before placing it into the STM for observation. We observed the formation of one-dimensional, defect- and kink-free Pt nanowires with a cross section of only one atom and lengths up to hundreds of nanometers [17]. Subsequently, the sample was exposed to air and transferred to a lowtemperature STM. After annealing the sample at $1050 \mathrm{~K}$ for about $10 \mathrm{~s}$ the Pt nanowires pattern was recovered.

In Fig. 1 an STM image of such an array of selforganizing Pt nanowires is shown. The spacing between adjacent $\mathrm{Pt}$ nanowires is 4 times the surface lattice constant of $\mathrm{Ge}(001)$, i.e., $1.6 \mathrm{~nm}$. We have performed scanning tunneling spectroscopy on the Pt-nanowires arrays at 


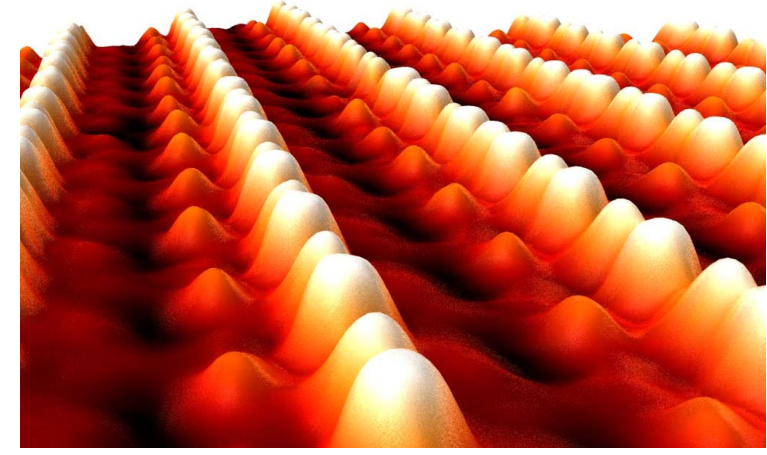

FIG. 1 (color online). Scanning tunneling microscopy image of an array of self-organizing Pt nanowires on $\mathrm{Ge}(001)$. Note the periodicity doubling of the Pt nanowires along the direction of elongation. The spacing between adjacent $\mathrm{Pt}$ nanowires is only $1.6 \mathrm{~nm}$. Sample bias $-1.35 \mathrm{~V}$ and tunneling current $0.54 \mathrm{nA}$.

both 77 and 300 K. Figure 2(a) shows the (spatially averaged) derivative $d I / d V$ recorded, at 77 and at $300 \mathrm{~K}$, on an array of Pt nanowires spaced $1.6 \mathrm{~nm}$ apart. The existence of a band gap near the Fermi level at $77 \mathrm{~K}$ as well as at $300 \mathrm{~K}$, in conjunction with the periodicity doubling of Pt nanowires along the germanium substrate dimer row direction, suggests a Peierls instability. In our present experimental setup we were not able to definitively settle this issue. STM measurements reveal that this periodicity doubling of the $\mathrm{Pt}$ nanowires persists up to at least $400 \mathrm{~K}$.

Figure 2(b) shows the local density of states (LDOS, i.e., the normalized derivative $(d I / d V) /(I / V))$ of the bare underlying terrace (lower curve) and of an array of $\mathrm{Pt}$ nanowires that are $1.6 \mathrm{~nm}$ (middle curve) or $2.4 \mathrm{~nm}$ (upper curve) spaced apart. All spectra are recorded at $77 \mathrm{~K}$. Note that the "bare underlying terrace", the so-called $\beta$ terrace, is not a clean $\mathrm{Ge}(001)$ surface, but is actually "platinum modified" (see Ref. [17] for more details). It is commonly accepted that the $(d I / d V) /(I / V)$ curve provides a better measure of the LDOS than the direct derivative $d I / d V$ [21-23]. In the middle $(d I / d V) /(I / V)$ curve in Fig. 2(b) two peaks are resolved. Most noticeable is the appearance of a peak at $77 \mathrm{~K}$ at $\sim 0.1 \mathrm{eV}$ above the Fermi level in the $(d I / d V) /(I / V)$ curve, which is not present in the room temperature data (note that this peak shifts to $\sim 0.15 \mathrm{~V}$ in the $d I / d V$ curve). Another peak positioned just below the Fermi level is present in the local density of states at $77 \mathrm{~K}$. The latter peak cannot be the metallic peak that has recently been ascribed to the symmetric appearing dimers on the clean $\mathrm{Ge}(001)$ at room temperature [20]. This specific metallic peak gradually fades away if the temperature of the $\mathrm{Ge}(001)$ substrate is lowered to temperatures below $\sim 200 \mathrm{~K}[24,25]$. Scanning tunneling spectroscopy experiments [Fig. 2(b) lower curve] reveal that the present novel metallic peak actually originates from the underlying $\beta$ terrace on which the Pt nanowires can form. In Ref. [17] it is suggested that these $\beta$ terraces consist out of pure Ge-Ge as well as mixed Ge-Pt dimers in a 1:1 ratio.

The novel electronic state located just above the Fermi level can be ascribed to either the Pt nanowires or to the troughs between the Pt nanowires. In order to identify the exact origin of this one-dimensional peak we have re-
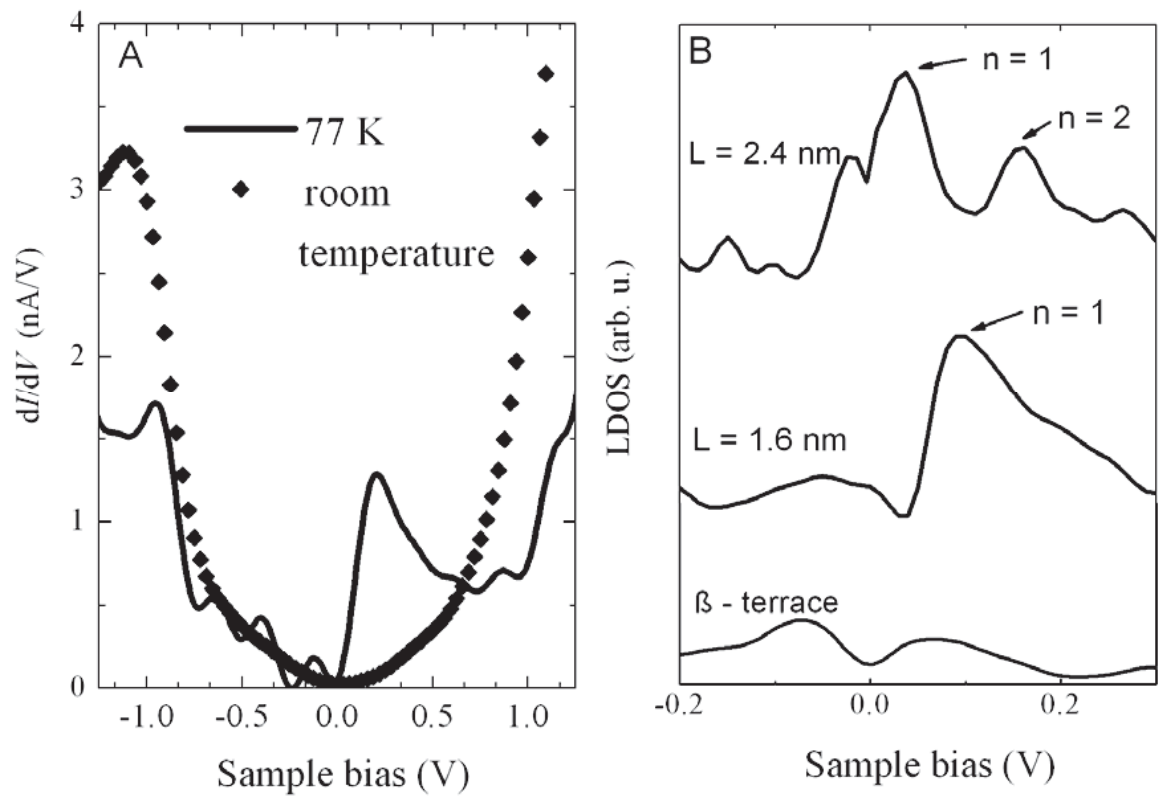

FIG. 2. (a) Differential conductivity $(d I / d V)$ of Pt-nanowires array at $300 \mathrm{~K}$ (diamonds) and $77 \mathrm{~K}$ (solid line). The Pt nanowires are $1.6 \mathrm{~nm}$ spaced apart. (b) Local density of states $((d I / d V) /(I / V))$ of the bare $\beta$ terrace (lower curve), troughs between Pt nanowires that are $1.6 \mathrm{~nm}$ spaced apart (middle curve) and troughs between Pt nanowires that are $2.4 \mathrm{~nm}$ spaced apart. All spectra are recorded at $77 \mathrm{~K}$. For $L=1.6 \mathrm{~nm}$ only one novel peak at $0.1 \mathrm{eV}$ above the Fermi level is observed, whereas for $L=2.4 \mathrm{~nm}$ two novel peaks are found at 0.04 and $0.16 \mathrm{eV}$ above the Fermi level, respectively. 


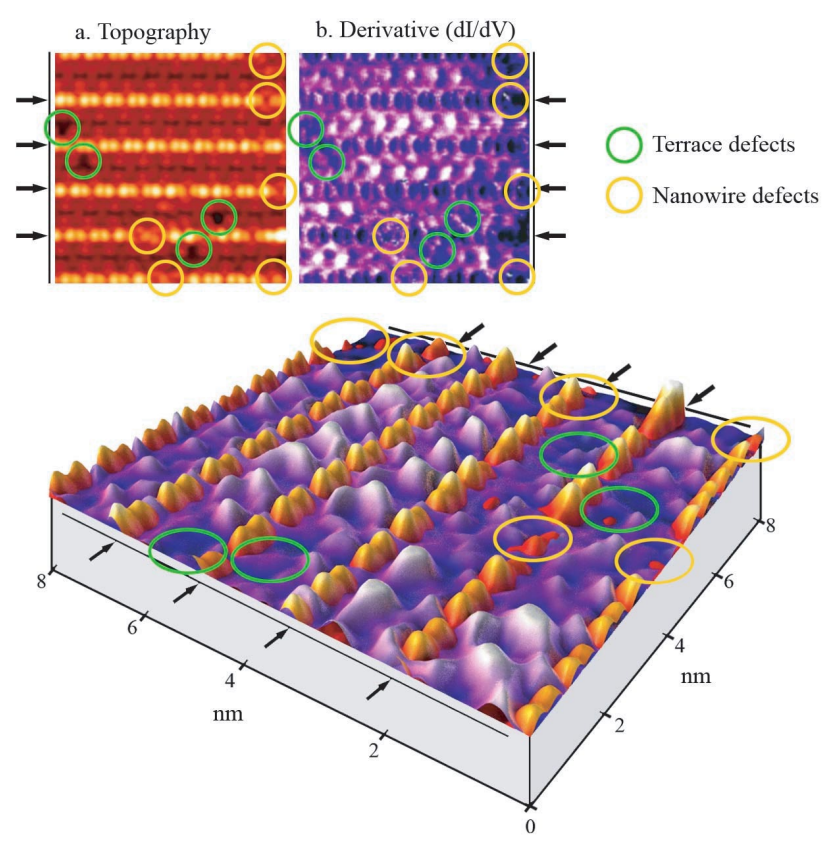

FIG. 3 (color). Topography (a) and spatial map of the differential conductivity $(d I / d V)$ (b) of a $8 \times 8 \mathrm{~nm}$ area with several Pt nanowires recorded at $T=77 \mathrm{~K}$. The sample bias is $0.15 \mathrm{~V}$ and the tunnel current is $0.437 \mathrm{nA}$. The $d I / d V$ map is recorded with a modulation voltage of $10 \mathrm{mV}$ and an oscillation frequency of $797 \mathrm{~Hz}$ (this frequency exceeds the bandwidth of the feedback loop). In the lower image a 3-dimensional representation of the topography (orange) and $d I / d V$ map (purple) is shown. The onedimensional electronic state is exclusively located in the troughs of the Pt nanowires (black arrows refer to the position of the Pt nanowires). The yellow and green circles (ellipses) refer to defects in the Pt nanowire and underlying substrate, respectively. The confinement of the electronic state disappears near these defects, as expected.

corded spatial maps of the derivative $d I / d V$ at $0.15 \mathrm{~V}$. $d I / d V$ maps were measured through lock-in detection of the ac tunnel current driven by a $797 \mathrm{~Hz}, 10 \mathrm{mV}$ (rms) signal added to the junction bias. From the spatial map presented in Fig. 3 it is immediately clear that this peak originates from the troughs and not from the Pt nanowires. We attribute the novel one-dimensional electronic state to confinement of the electronic surface state near the Fermi level. The apparent barrier height above a local topographic protrusion in the nanowires is larger, i.e., the effective decay length is smaller, than above a local topographic depression. This spatial variation in the transmission coefficient will show up in spatially resolved measurements of $d I / d V$ as a "background" that is essentially an inverted constant current topography. The observation that missing atoms in the troughs result in a local decrease of the $d I / d V$ signal implies that the latter effect is of minor importance (see the four green circles and ellipses in Fig. 3). The shift in energy of the one-dimensional state with respect to the two-dimensional surface state is con- vincingly attributed to the confinement of the electrons between neighboring Pt nanowires. Near defects of the nanowire the peak position is either shifted towards higher energy (in case the defect narrows the trough) or lower energy (in case the defect widens the trough). Typically the peak shifts decay over a distance of a few nanometers measured along the trough direction [26].

Bearing in mind that the spacing between adjacent $\mathrm{Pt}$ nanowires is $1.6 \mathrm{~nm}$ we calculate, using the solutions of a quantum mechanical particle in a box, an energy shift of $0.147 \mathrm{eV}$, which is only slightly higher than our observations. Additional subbands (e.g., $n=2$ at $\sim 0.4 \mathrm{eV}$ and $n=3$ at $\sim 0.9 \mathrm{eV})$ are, however, not observed. This is most probably due to a finite barrier height. This will also result in a renormalization of the energy position of all subbands.

Besides the majority of the Pt nanowires that are $1.6 \mathrm{~nm}$ spaced apart, we have also identified a few that are $2.4 \mathrm{~nm}$ spaced apart. The latter actually form as light domain walls between the $(4 \times 2)$ domains established by the Pt nanowires. Sure enough, the corresponding density of states shows not only one single peak, as for the $1.6 \mathrm{~nm}$ spacing, but rather two peaks at about 0.04 and $0.16 \mathrm{eV}$ [see Fig. 2(b)] [27]. As we will point out below, this demonstrates that indeed we are dealing with the confinement of a regular surface state between the Pt chains, i.e., the linearly arranged $\mathrm{Pt}$ atoms provide a one-dimensional confining potential. In contrast to $n=1$ states, which exhibit a maximum in the $d I / d V$ signal in the middle of the well, the $n=2$ state exhibits a node in the middle of the well. In fact, we have a textbook example of the simple quantum mechanical problem of a quantum mechanical particle trapped in a well of finite depth. For the sake of simplicity we first assume that the well is infinitely deep. The energy positions are given by

$$
E=\frac{\hbar^{2} \pi^{2}}{2 m}\left(\frac{n}{L}\right)^{2}
$$

where $\hbar$ is Planck's constant, $m$ the mass of the electron, and $L$ the width of the well. The three novel electronic states at $0.10 \mathrm{eV}(L=1.6 \mathrm{~nm}$ and $n=1), 0.04$ and $0.16 \mathrm{eV}$ $(L=2.4 \mathrm{~nm}$ and $n=1,2)$ obey the $(n / L)^{2}$ scaling law amazingly well. Next we consider a slightly more sophisticated model: we assume a finite square well with depth $V_{0}$, an effective well width $L_{\text {eff }}\left(=n L_{0}\right.$, where $L_{0}=1.6$ or $2.4 \mathrm{~nm})$ and an effective mass of the electrons $\left(m_{\mathrm{eff}}=\right.$ $\lambda m_{0}$, where $m_{0}$ is the rest mass of the electron). In Gasiorowicz [28] this problem is treated extensively. The solutions are

$$
\begin{aligned}
& \frac{\sqrt{\gamma-y^{2}}}{y}=\tan y \quad \text { (even solutions) } \\
& \frac{\sqrt{\gamma-y^{2}}}{y}=-\cot y \quad \text { (odd solutions) }
\end{aligned}
$$


where $\gamma=\frac{2 m_{\text {eff }} V_{0} L_{\text {eff }}^{2}}{\hbar^{2}}, y=L_{\text {eff }} \sqrt{\frac{2 m_{\text {eff }} E}{\hbar^{2}}}$ and $E$ is the energy of the quantum mechanical particle [28]. We find that all three energy levels can be fitted very well for $V_{0}=0.21 \mathrm{eV}$ and $\nu \sqrt{\lambda}=0.6$. Assuming for instance $\nu=0.8$ results in an effective mass of $0.56 m_{0}$. Unfortunately, the relatively low value of $V_{0}$ does not allow confined energy levels higher than $n=1 \quad(L=1.6 \mathrm{~nm})$ and $n=2(L=2.4 \mathrm{~nm})$. Indeed, this consequence is perfectly consistent with the experiment since all these energy levels with $n>1(L=$ $1.6 \mathrm{~nm})$ and $n>2(L=2.4 \mathrm{~nm})$ are absent.

We have presented a study of the electronic properties of self-organized Pt-nanowires arrays at low temperatures on $\mathrm{Ge}(001)$. We have discovered the existence of a set of novel electronic states at $77 \mathrm{~K}$, which are absent at room temperature. The energy of the novel electronic states are $0.10 \mathrm{eV}(n=1$ and $L=1.6 \mathrm{~nm}), 0.04$ and $0.16 \mathrm{eV}(n=$ 1,2 and $L=2.4 \mathrm{~nm}$ ) higher than that of a twodimensional surface state on the Pt-modified $\beta$ terraces, which is located near the Fermi level. A careful analysis of our differential conductivity data reveals that these states are actually located within the troughs between the $\mathrm{Pt}$ nanowires. The increase in energy is a result of the confinement of the nearly free surface state electrons between the Pt nanowires and its values agree well with basic theoretical estimates. Finally, the fact that the high density of states $(0.1 \mathrm{eV})$ at the center of the troughs gradually fades away near defects in either the Pt nanowires or the underlying terrace provides strong additional proof in favor of our assignment of the electronic state to quantum confinement of electrons in between Pt nanowires.

This work is financially supported by the Stichting voor Fundamenteel Onderzoek der Materie (FOM, 03PR2208)) and Nederlandse Organisatie voor Wetenschappelijk Onderzoek (NWO, VIDI TTF 5847).

*Corresponding author. Email address: h.j.w.zandvliet@utwente.nl

[1] N. Agraï, A. L. Yeyati, and J. M. van Ruitenbeek, Phys. Rep. 377, 81 (2003), and references therein.

[2] H. W. Yeom et al., Phys. Rev. Lett. 82, 4898 (1999).

[3] A. Yacoby, H. L. Stormer, N. S. Wingreen, L. N. Pfeiffer, K. W. Baldwin, and K. W. West, Phys. Rev. Lett. 77, 4612 (1996).

[4] P. Gambardella, A. Dallmeyer, K. Maiti, M. C. Malagoli, W. Eberhardt, K. Kern, and C. Carbone, Nature (London) 416, 301 (2002).
[5] P. Gambardella, A. Dallmeyer, K. Maiti, M. C. Malagoli, S. Rusponi, P. Ohresser, W. Eberhardt, C. Carbone, and K. Kern, Phys. Rev. Lett. 93, 077203 (2004).

[6] S. Tarucha, T. Honda, and T. Saku, Solid State Commun. 94, 413 (1995).

[7] Z. Yao, H. W. C. Postma, L. Balents, and C. Dekker, Nature (London) 402, 273 (1999).

[8] Nanostructures and Mesoscopic Systems, edited by W.P. Kirk and M. A. Reed (Academic Press, New York, 1992).

[9] Physics and Technology of Submicron Structures, edited by H. Heinrich, G. Bauer, and F. Kuchar (Springer, Berlin, 1988).

[10] M.F. Crommie, C.P. Lutz, and D. M. Eigler, Nature (London) 363, 524 (1993).

[11] M. F. Crommie, C. P. Lutz, and D. M. Eigler, Science 262, 218 (1993).

[12] L. Bürgi, O. Jeandupeux, A. Hirstein, H. Brune, and K. Kern, Phys. Rev. Lett. 81, 5370 (1998).

[13] O. Sánchez, J. M. García, P. Segovia, J. Alvarez, A. L. Vázquez de Parga, J.E. Ortega, M. Prietsch, and R. Miranda, Phys. Rev. B 52, 7894 (1995).

[14] J. Li, W.-D. Schneider, R. Berndt, and S. Crampin, Phys. Rev. Lett. 80, 3332 (1998).

[15] N. Nilius, T. M. Wallis, and W. Ho, Science 297, 1853 (2002).

[16] T. M. Wallis, N. Nilius, and W. Ho, Phys. Rev. Lett. 89, 236802 (2002).

[17] O. Gurlu, A. O. A. Adam, H. J. W. Zandvliet, and B. Poelsema, Appl. Phys. Lett. 83, 4610 (2003).

[18] H. J. W. Zandvliet, B. S. Swartzentruber, W. Wulfhekel, B. J. Hattink, and B. Poelsema, Phys. Rev. B 57, R6803 (1998).

[19] H. J. W. Zandvliet, Phys. Rep. 388, 1 (2003).

[20] O. Gurlu, H. J. W. Zandvliet, and B. Poelsema, Phys. Rev. Lett. 93, 066101 (2004).

[21] J. A. Stroscio, R. M. Feenstra, and A. P. Fein, Phys. Rev. Lett. 57, 2579 (1986)

[22] R. M. Feenstra, J. A. Stroscio, and A.P. Fein, Surf. Sci. 181, 295 (1987).

[23] N. D. Lang, Phys. Rev. B 34, 5947 (1986).

[24] S. D. Kevan and N. G. Stoffel, Phys. Rev. Lett. 53, 702 (1984).

[25] S. D. Kevan, Phys. Rev. B 32, 2344 (1985).

[26] H. J. W. Zandvliet (unpublished).

[27] The broadening of the peaks depends on the thermal energy $k T(6.6 \mathrm{meV})$, the modulation voltage $V_{\text {mod }}$ (10 meV), and the intrinsic peak width. The latter is, however, unknown.

[28] S. Gasiorowicz, in Quantum Physics (John Wiley \& Sons, New York, 1995), p. 75. 